\title{
Effect of long-range Coulomb interaction on shot-noise suppression in ballistic transport
}

\author{
T. González, O. M. Bulashenko,* J. Mateos, and D. Pardo \\ Departamento de Física Aplicada, Universidad de Salamanca, Plaza de la Merced s/n, E-37008 Salamanca, Spain \\ L. Reggiani \\ Istituto Nazionale di Fisica della Materia, Dipartimento di Scienza dei Materiali, Università di Lecce Via Arnesano, 73100 Lecce, Italy
}

(Received 19 December 1996)

\begin{abstract}
We present a microscopic analysis of shot-noise suppression due to long-range Coulomb interaction in semiconductor devices under ballistic transport conditions. An ensemble Monte Carlo simulator selfconsistently coupled with a Poisson solver is used for the calculations. A wide range of injection-rate densities leading to different degrees of suppression is investigated. A sharp tendency of noise suppression at increasing injection densities is found to scale with a dimensionless Debye length related to the importance of spacecharge effects in the structure. [S0163-1829(97)09735-X]
\end{abstract}

The phenomenon of shot noise, associated with the randomness in the flux of carriers crossing the active region of a device, has become a fundamental issue in the study of electron transport through mesoscopic devices. In particular, the possibility of shot-noise suppression has recently attracted a lot of attention, both theoretically and experimentally. ${ }^{1}$ At low frequency (small compared to the inverse transit time through the active region) the power spectral density of shot noise is given by $S_{I}=\gamma 2 q I$, where $I$ is the dc current, $q$ is the electron charge, and $\gamma$ is the suppression factor. When the carriers crossing the active region are uncorrelated, full shot noise with $\gamma=1$ (Poisson statistics) is observed. However, correlations between carriers can reduce the shot-noise value, giving $\gamma<1$. In real mesoscopic devices different types of mechanisms resulting in shot-noise suppression can be distinguished: (i) statistical correlations due to the Pauli exclusion principle (important for degenerate materials obeying Fermi statistics), (ii) short-range Coulomb interaction (electron-electron scattering), and (iii) long-range Coulomb interaction (by means of the self-consistent electric potential). While the first two mechanisms have been extensively discussed in solid-state literature, ${ }^{1}$ the last one has received less attention, ${ }^{2}$ although its role in shot-noise suppression has been known for a long time in vacuum-tube devices. ${ }^{3}$ The only exception that should be mentioned is the Coulomb blockade in resonant-tunneling devices, which can be also referred to as the last mechanism of suppression. The blockade is provided by a built-in charge inside a quantum well which redistributes the chemical potential, and prevents the incoming carriers from passing through the well, thereby resulting in carrier correlation and shot-noise suppression (see the experimental evidence ${ }^{4}$ ). The Coulomb blockade is a consequence of long-range Coulomb interaction, and it acts under the sequential tunneling regime of carrier transport.

The main objective of the present paper is to prove the importance of long-range Coulomb interaction between the carriers on the shot-noise power spectrum under the ballistic regime of electron transport. The ballistic regime is now accessible in modern mesoscopic devices like electron waveguides, quantum point contacts, etc., which have characteristic lengths of the order, or smaller, than the carrier mean free path. The current existing theories invoked to interpret the experimentally observed shot-noise suppression in such devices $^{5-7}$ assume that carriers move inside the device without inducing any redistribution of the electric potential. We use a more rigorous approach which includes long-range Coulomb interaction between the carriers by considering the carrier transport in the self-consistent potential governed by the Poisson equation. We show that under the ballistic regime this interaction is crucial, and that noise characteristics are strongly modified depending on whether the carrier correlation (mediated by the field) is taken into account or not.

To this purpose we consider a simple structure: a lightly doped active region of a semiconductor device sandwiched between two heavily doped contacts injecting the carriers into the active region. The device then acts similarly to a vacuum diode, with a relevant difference in the fact that there are two opposing currents instead of a single current. Electrons are emitted from the contacts according to a thermal-equilibrium Maxwell-Boltzmann distribution, and they move ballistically inside the active region (the mean free path is considered to be much larger than the distance $L$ between the contacts) according to the semiclassical equations of motion. The fluctuating emission rate at the contacts is taken to follow a Poisson statistics. This means that the time between two consecutive electron emissions is generated according to the probability density $P(t)=\Gamma e^{-\Gamma t}$, where $\Gamma=\frac{1}{2} n_{c} v_{\text {th }} S$ is the injection rate, with $n_{c}$ the electron density at the contact, $S$ the cross sectional area of the device, and $v_{\mathrm{th}}=\sqrt{2 k_{B} T /(\pi m)}$ the thermal velocity ( $T$ is the lattice temperature, $k_{B}$ Boltzmann constant, and $m$ the electron effective mass). The electron gas is assumed to be nondegenerate to exclude possible correlations due to the Fermi statistics. For simplicity, the conduction band of the semiconductor is considered to be spherically parabolic. Both the time-averaged current and the current fluctuations inside the active region of the device are analyzed for different bias voltages applied between the contacts. The calculations are performed by using an ensemble Monte Carlo simulator selfconsistently coupled with a Poisson solver (PS). By using this approach we can analyze much more general situations than those studied in previous analytical calculations. ${ }^{2}$ 
For the calculations we used the following set of parameters: $T=300 \mathrm{~K}, m=0.25 m_{0}$, dielectric constant $\varepsilon=11.7 \varepsilon_{0}$, sample length $L=2000 \AA$, and contact doping $n_{c}$ ranging between $10^{13}$ and $4 \times 10^{17} \mathrm{~cm}^{-3}$ (always at least two orders of magnitude higher than the sample doping). However, we must stress that the results we are going to present do not depend on the particular values of these parameters, but only on the dimensionless length $\lambda=L / L_{D c}$, where $L_{D c}=\sqrt{\varepsilon k_{B} T / q^{2} n_{c}}$ is the Debye length corresponding to the carrier concentration at the contact.

Let us discuss briefly the steady-state spatial distributions of the quantities of interest inside the sample. In a general case the carrier concentration is nonuniform, having maximum values at the contacts due to the electron injection and decaying toward the middle of the sample. Accordingly, without an external voltage bias the potential distribution has a minimum in the middle of the sample due to the space charge. When a positive voltage is applied to the anode, the minimum is displaced toward the cathode, while its amplitude tends to diminish. This minimum provides a potential barrier for the electrons moving between the contacts, so that a part of the electrons, not having enough energy to go over the barrier, are reflected back to the contacts. The most important fact is that the transmission through the barrier is current dependent, which is crucial in calculating the noise characteristics. In the structure the current is limited by the space charge and increases linearly with the applied voltage up to a certain value of the external bias when the barrier vanishes, so that all the electrons emitted from the cathode can reach the anode. Under the latter regime the current is saturated and becomes independent of the bias. ${ }^{8}$ It is important to stress that in our approach we do not impose a fixed number of electrons $N$ to be present inside the sample. The value of $N$ is determined by the emission rates of the contacts and the applied bias. Therefore, $N$ fluctuates in time and we can evaluate both the time-averaged value $\langle N\rangle$ and its fluctuations by means of the Monte Carlo algorithm. One can observe that $\langle N\rangle$ is constant at the increasing part of the current-voltage characteristic, and it decreases with the bias once the current is saturated.

Under a fixed applied voltage the current density in the structure is given by $I(t)=(q / L) \sum_{i=1}^{N(t)} v_{i}(t)$, where $v_{i}(t)$ is the instantaneous velocity component along the field direction of the $i$ th particle. ${ }^{9}$ The current autocorrelation function $C_{I}(t)=\left\langle\delta I\left(t^{\prime}\right) \delta I\left(t^{\prime}+t\right)\right\rangle$ is evaluated from the sequence $I(t)$ obtained from the Monte Carlo simulation, where the current fluctuation is given by $\delta I(t)=I(t)-\langle I\rangle$. To clarify the role of different contributions to the current noise we decompose the current autocorrelation function into three main contributions $C_{I}(t)=C_{V}(t)+C_{N}(t)+C_{V N}(t)$ associated, respectively, with the fluctuations in the mean velocity of electrons $C_{V}$, the fluctuations in the carrier number $C_{N}$, and the velocity-number cross correlation $C_{V N}$. The corresponding formulas are given by ${ }^{9}$

$$
\begin{gathered}
C_{V}(t)=\frac{q^{2}}{L^{2}}\langle N\rangle^{2}\left\langle\delta v\left(t^{\prime}\right) \delta v\left(t^{\prime}+t\right)\right\rangle, \\
C_{N}(t)=\frac{q^{2}}{L^{2}}\langle v\rangle^{2}\left\langle\delta N\left(t^{\prime}\right) \delta N\left(t^{\prime}+t\right)\right\rangle,
\end{gathered}
$$

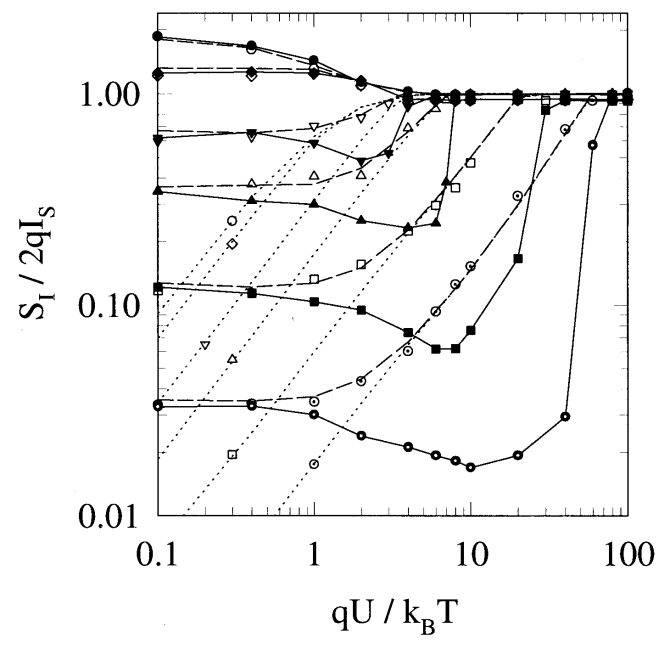

FIG. 1. Current-noise spectral density $S_{I}$ vs applied voltage $U$ calculated by using static (open symbols) and self-consistent (closed symbols, solid line) potentials for several injection-rate densities $n_{c}$ (in $\mathrm{cm}^{-3}$, with the corresponding $\lambda$ ): $(\bigcirc) 10^{13}, \lambda=0.15$; $(\diamond) 2 \times 10^{15}, \lambda=2.18 ;(\nabla) 10^{16}, \lambda=4.88 ;(\triangle) 2.5 \times 10^{16}, \lambda$ $=7.72$; $\square$ ) $10^{17}, \lambda=15.45$; $(\odot) 4 \times 10^{17}, \lambda=30.9$. The static case is shown to be nicely described by Eq. (2) (dashed line). The dotted lines represent $2 q I$ (marked for each injection-rate density by the corresponding symbol).

$$
C_{V N}(t)=\frac{q^{2}}{L^{2}}\langle v\rangle\langle N\rangle\left\langle\delta v\left(t^{\prime}\right) \delta N\left(t^{\prime}+t\right)+\delta N\left(t^{\prime}\right) \delta v\left(t^{\prime}+t\right)\right\rangle .
$$

Figure 1 shows the low-frequency value of the spectral density of current fluctuations $S_{I}=2 \int_{-\infty}^{\infty} C_{I}(t) d t$ normalized to $2 q I_{s}$, where $I_{s}=q \Gamma=\frac{1}{2} q n_{c} v_{\mathrm{th}} S$ is the saturation current (notice that this is the maximum current that a contact may provide). This normalization is performed in order to compare the results for different injection-rate densities (different contact dopings). We provide the results for two different simulation schemes. The first one involves a dynamic PS, which means that any fluctuation of space-charge appeared due to the random injection from the contacts causes a redistribution of the potential, which is self-consistently updated by solving the Poisson equation at each time step during the simulation to account for the fluctuations associated with long-range Coulomb interaction. In the second scheme we use a static PS to calculate only the stationary potential profile, and, once the steady state is reached, the PS is switched off, so that the carriers move in the frozen nonfluctuating electric field profile. We checked that both schemes give exactly the same steady-state spatial distributions and total current, but the noise characteristics are different. Several values of $n_{c}$ (and therefore several injection-rate densities) have been considered. As $n_{c}$ increases, space-charge effects become more and more significant, the dimensionless parameter $\lambda$ being the indicator of their importance.

In the static case, by increasing the applied voltage $U$ we always obtain an excellent coincidence with the well-known formula ${ }^{10}$ used to describe the crossover from thermal to shot noise when carrier correlation plays no role (represented in the figure by dashed lines): 

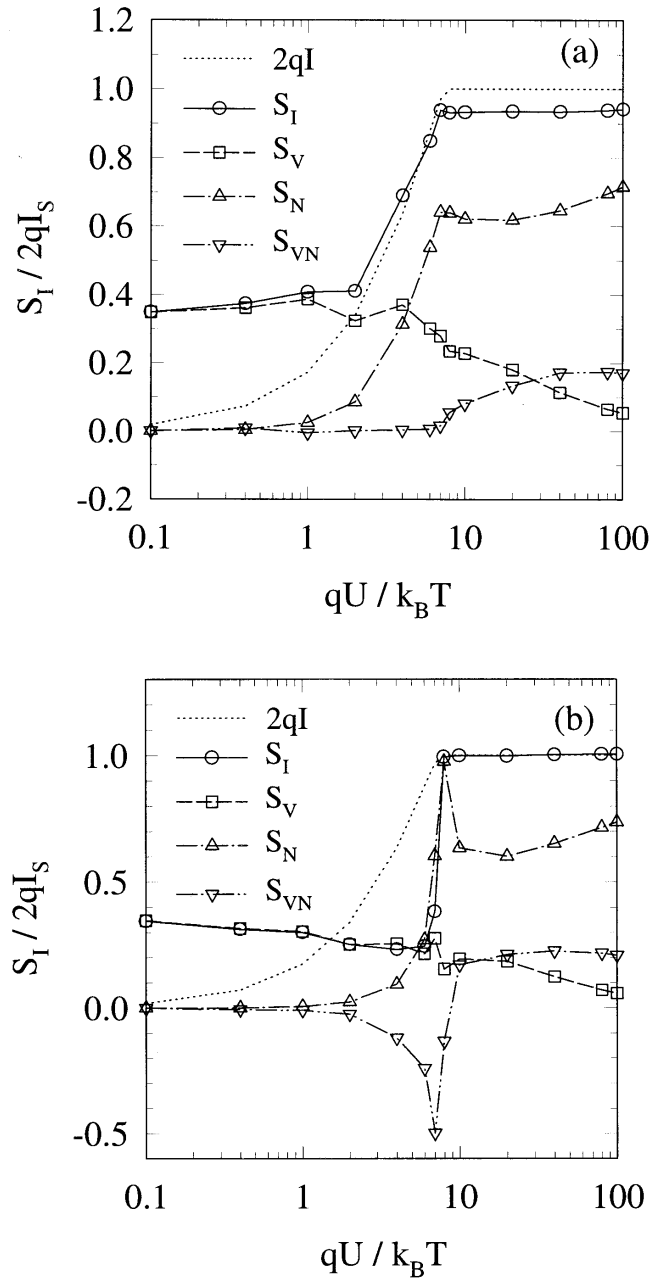

FIG. 2. Decomposition of the spectral density of current fluctuations $S_{I}$ into velocity, number, and velocity-number contributions vs applied voltage for the case $n_{c}=2.5 \times 10^{16} \mathrm{~cm}^{-3}, \lambda=7.72$ calculated by using (a) the static and (b) the dynamic Poisson solver.

$$
S_{I}=2 q\left(I^{+}+I^{-}\right)=2 q I \operatorname{coth}\left(q U / 2 k_{B} T\right),
$$

where $I=I^{+}-I^{-}$is the total current flowing through the diode, consisting of two opposing currents, $I^{+}=I_{s} \exp \left[-q V_{m} / k_{B} T\right]$ in the forward-bias direction and $I^{-}=I_{s} \exp \left[-q\left(V_{m}+U\right) / k_{B} T\right]$ in the opposite direction, $V_{m}$ being the potential minimum induced by the space-charge, which is dependent on $U$. This agreement supports the validity of the simulation scheme used for the calculations. For $q U \ll k_{B} T, \quad I^{+} \sim I^{-}, \quad$ thermal noise is dominant and $S_{I} \approx 4 q I_{s} \exp \left[-q V_{m} / k_{B} T\right]$. Therefore, for the lowest value of $\lambda$ (when space-charge is negligible and $V_{m} \rightarrow 0$ ), $S_{I} \rightarrow 4 q I_{s}$, while as $\lambda$ increases $V_{m}$ becomes significant and $S_{I}$ decreases. When $q U \geqslant k_{B} T, I^{+} \gg I^{-}$, the transition from thermal noise to shot noise takes place and $S_{I} \approx 2 q I^{+}$. Finally, for the highest values of $U$, saturation occurs, $V_{m}$ vanishes, and $S_{I} \approx 2 q I_{s}$.

For the lowest values of $\lambda$ no difference between the dynamic and static cases is obviously detected. However, for higher $\lambda$, when space-charge effects become significant, the picture is drastically different for the dynamic case. Starting from $q U \sim k_{B} T$ the current noise, instead of increasing, decreases until the proximity of saturation. Under saturation, the results for both schemes coincide and full shot noise $2 q I_{s}$ is recovered. When compared with the static case the noise suppression is stronger for higher $\lambda$ (more important spacecharge effects).

To understand the physical reason for the shot-noise suppression, in Fig. 2 we provide the decomposition of $S_{I}$, calculated with static and dynamic PS, into the additive contributions $S_{V}, S_{N}$, and $S_{V N}$ [Eqs. (1)] for different applied voltages $U$ and $\lambda=7.72\left(n_{c}=2.5 \times 10^{16} \mathrm{~cm}^{-3}\right)$. The contributions of $S_{N}$ and $S_{V N}$ to the current noise vanish at equilibrium $(U \rightarrow 0)$, since they are proportional to $\langle v\rangle^{2}$ and $\langle v\rangle \rightarrow 0$. Thus for small biases $\left(q U \ll k_{B} T\right) S_{I} \sim S_{V}$, which means that the current noise is thermal noise associated with velocity fluctuations and is governed by the Nyquist theorem $S_{I} \approx 4 k_{B} T G$, with $G=d I /\left.d V\right|_{V=0}$ the conductance. For this case the results for the static [Fig. 2(a)] and dynamic [Fig. 2(b)] schemes evidently coincide. However, starting from $q U \sim k_{B} T$ the difference becomes drastic. For the dynamic case the velocity-number correlations, represented by $S_{V N}$, are negative, while for the static case they are positive. Furthermore, for the current fluctuations calculated using the self-consistent potential, $S_{N}$ and $S_{V N}$ are of opposite sign and compensate for each other, so that $S_{I}$ approximately follows $S_{V}$ as long as the current is space-charge limited. As a consequence, the current noise, which now corresponds to shot noise, is considerably suppressed below the value $2 q I$ given by the static case. This result reflects the fact that as the carriers move through the active region, the dynamic fluctuations of the electric field modulate the transmission through the potential minimum and smooth the current fluctuations imposed by the random injection at the contacts. Therefore, the coupling between number and velocity fluctuations induced by the self-consistent potential fluctuations is mainly responsible, through $S_{V N}$, for the shot-noise suppression. This velocity-number coupling becomes especially pronounced just before the current saturation $\left(U \approx 7 k_{B} T / q\right)$, when the potential minimum is close to vanishing completely $\left(V_{m} \rightarrow 0\right.$ ), and the fluctuations of the potential barrier modulate the transmission of the more populated states of the injected carriers (the low-velocity states). Under saturation

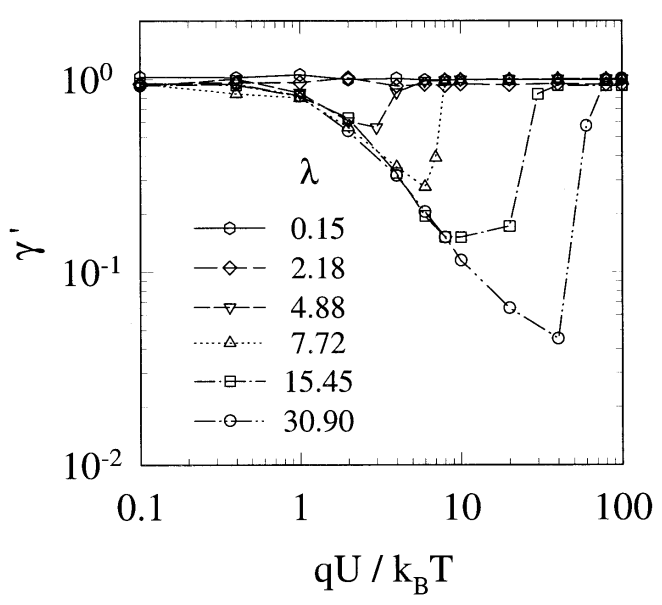

FIG. 3. Shot noise reduction factor $\gamma^{\prime}$ vs voltage $U$ for several injection-rate densities $n_{c}$ (different values of $\lambda$ ). 
conditions space-charge effects do not modulate the random injection (no potential minimum is present), and again both dynamic and static cases provide the same additive contributions and total noise $\left(2 q I_{s}\right)$.

Finally, in Fig. 3 we present the reduction factor $\gamma^{\prime}$ defined as the ratio between $S_{I}$ as calculated with the dynamic PS $\left(S_{I-d}\right)$ and, as given by Eq. (2) neglecting the influence of long-range Coulomb interaction, $\gamma^{\prime}=S_{I-d} /$ $\left[2 q I \operatorname{coth}\left(q U / 2 k_{B} T\right)\right]$. In the context of our calculations $\gamma^{\prime}$ is more appropriate than the standard suppression factor $\gamma=S_{I-d} /(2 q I)$, since it covers both the thermal and shotnoise range of applied voltages. Here it is observed how the shot-noise suppression becomes more pronounced as $\lambda$ increases. For example, for $\lambda=30.9$ it reaches 0.04 . Thus our self-consistent approach predicts much lower values of the suppression factor than the previous analytical model of van der Ziel and Bosman, ${ }^{2}$ where the dependence of the potential minimum and its position on the applied voltage was not taken into account.

In principle, the value of the parameter $\gamma^{\prime}$ in our model has no lower limit. We observe that it follows asymptotically the behavior $\gamma^{\prime} \sim k_{B} T / q U$ in the range where shot-noise suppression is more pronounced $\left(q U \gg k_{B} T, U \ll U_{\text {sat }}\right) . \gamma^{\prime}$ can reach a value as low as desired by appropriate increasing the sample length and/or the carrier concentration at the contact, provided the transport remains ballistic. However, with increasing device length (or lattice temperature) the carrier transport actually goes from the ballistic to the diffusive regime, and the shot-noise suppression is washed out. Therefore, the maximum suppression factor predicted by our calculations for a system with a given value of the mean free path $\lambda_{p}$ would be obtained approximately at $\lambda_{\max } \sim \lambda_{p} / L_{D c}$. Moreover, when the carrier concentration at the contact is increased so that the electron gas becomes degenerate, statistical (Pauli) correlations between the carriers appear, which will be additive (in the sense of shot-noise suppression) to the Coulomb correlations.
It should be emphasized that two essential conditions are necessary for the strong shot-noise suppression due to longrange Coulomb interaction: (i) the presence of a potential barrier inside the device which controls the current, and (ii) the carrier transmission through the barrier should depend on the current. This fact is quite general and, therefore, the results obtained in the present paper extend to much more physical situations. For example, in a recent experiment by Reznikov et al. ${ }^{6}$ the shot-noise level measured in a quantum point contact in the pinched-off regime was found to be unexpectedly low (about one-third and less). In that regime the transport is controlled by the potential barrier present at the gates, and both conditions for the shot-noise suppression mentioned above are fulfilled. Hence the results obtained in our calculations strongly support the suggestion of the authors of the experiment that the origin of the discrepancies between experimental results and theoretical predictions lies in the disregarding of Coulomb interaction between electrons passing through the contact. More precisely, the electron flow considerably modifies the potential distribution inside the contact, yielding the coupling of velocity-number fluctuations, and resulting in shot-noise suppression.

In conclusion, we have investigated the influence of longrange Coulomb interaction on shot-noise suppression in ballistic transport by using an ensemble Monte Carlo simulator self-consistently coupled with a Poisson solver. We have found that this suppression is stronger as space-charge effects become more important, and it can be monitored by a dimensionless parameter $\lambda$. More than one order of magnitude of shot-noise suppression is predicted. The main contribution to the suppression is found to originate from the velocity-number correlations induced by the self-consistent field.

This work has been partially supported by the Comisión Interministerial de Ciencia y Tecnología through Project No. TIC95-0652.
*Present address: Dept. Física Fonamental, Universitat de Barcelona, Av. Diagonal 647, E-08028 Barcelona, Spain.

${ }^{1}$ See, e.g., recent review M. J. M. de Jong and C. W. J. Beenakker, cond-mat/9611140 (unpublished).

${ }^{2}$ A. van der Ziel and G. Bosman, Phys. Status Solidi A 73, K93 (1982).

${ }^{3}$ D. O. North, RCA Rev. 4, 441 (1940); 5, 106 (1941).

${ }^{4}$ H. Birk, M. J. M. de Jong, and C. Schönenberger, Phys. Rev. Lett. 75, 1610 (1995).

${ }^{5}$ Y. P. Li, D. C. Tsui, J. J. Heremans, J. A. Simmons, and G. W. Weimann, Appl. Phys. Lett. 57, 774 (1990).
${ }^{6}$ M. Reznikov, M. Heiblum, H. Shtrikman, and D. Mahalu, Phys. Rev. Lett. 75, 3340 (1995).

${ }^{7}$ A. Kumar, L. Saminadayar, D. C. Glattli, Y. Jin, and B. Etienne, Phys. Rev. Lett. 76, 2778 (1996).

${ }^{8}$ This behavior contrasts with the exponential and Child-Langmuir $I-V$ dependences typical of vacuum diodes. It is due to the presence of the opposite current injected at the anode.

${ }^{9}$ T. González and D. Pardo, J. Appl. Phys. 73, 7453 (1993).

${ }^{10}$ A. van der Ziel, Noise in Solid State Devices and Circuits (Wiley, New York, 1986). 